\title{
REAL TIME SOLUTION OF NONLINEAR FILTERING PROBLEM WITHOUT MEMORY I
}

\author{
Shing-Tung YAU* And Stephen S.-T. YAU*†
}

\section{Introduction}

The nonlinear filtering problem involves the estimation of a stochastic process $x=\left\{x_{t}\right\}$ (called the signal or state process) that cannot be observed directly. Information containing $x$ is obtained from observations of a related process $y=$ $\left\{y_{t}\right\}$ (the observation process). The goal of nonlinear filtering is to determine the conditional expectations of the form $E\left[\phi\left(x_{t}\right): y_{s}, 0 \leq s \leq t\right]$, or perhaps even the computation of the entire conditional density $\rho(t, x)$ of $x_{t}$ given the observation history $\left\{y_{s}, 0 \leq s \leq t\right\}$. When the observations are received sequentially, as in many practical applications, it is preferable that this computation be performed recursively in terms of a statistic $\theta=\left\{\theta_{t}\right\}$, which can be updated by using only the latest observations

$$
\theta_{t+\tau}=\alpha\left(t, \tau, \theta_{t},\left\{y_{s}, t \leq s \leq t+\tau\right\}\right)
$$

and from which estimates can be calculated in a "pointwise" or "memoryless" manner:

$$
E\left[\phi\left(x_{s}\right): y_{s}, 0 \leq s \leq t\right]=\beta\left(t, y_{t}, \theta_{t}\right) .
$$

In many cases, $\theta_{t}$ is computable with a finite set of differential equations driven by $y$. In these cases, the practical implication of recursiveness is the possible implementation of the filter (1.1) - (1.2) in real time. We refer the readers to the excellent expository article by Marcus [Ma] for details. Mathematically, the unnormalized conditional density satisfies a time-varying parabolic partial differential equation (DMZ equation) driven by observation process y (cf. (2.5)). One would like to solve this DMZ equation by means of the solution of a partial differential equation which is independent of observation $y$ and the solution of a finite system of ordinary differential equations driven by $y$. Indeed, in our previous paper [Ya-Ya1], we have found the solution of DMZ equation for linear filtering system and exact filtering system with arbitrary initial condition in terms of the solution of Kolmogorov equation (independent of observation $y$ ) and the solution of a finite system of linear ordinary differential equation driven

Received May 30, 2000.

*Research partially supported by U.S. Army Research Office: DAAD19-99-1-0203.

${ }^{\dagger}$ Research partially supported by NSF Grant. 
by $y$. More recently, $\mathrm{Hu}$ and $\mathrm{Yau}[\mathrm{Hu}-\mathrm{Ya}]$ have done the same thing for the so-called Yau filtering in the sense of Chen [Ch].

Historically Kalman-Bucy first established the finite dimensional filters for linear filtering system with Gaussian initial distribution in 1961. Ever since the technique of the Kalman-Bucy filters was popularized, there has been an intense interest in finding new classes of finite dimensional recursive filters. In the sixties and early seventies, the basic approach to nonlinear filtering theory was via the "innovations method" originally proposed by Kailath and subsequently rigorously developed by Fujisaki, Kallianpur, and Kunita in 1972. As pointed out by Mitter, the difficulty with this approach is that the innovations process is not, in general, explicitly computable. In view of this weakness, Brockett, and Mitter proposed independently the idea of using estimation algebras to construct finite-dimensional nonlinear filters. The idea is to imitate the WeiNorman approach of using the Lie algebraic method to solve the DMZ equation, which the unnormalized conditioned probability density of the state $x_{t}$ must satisfy. This Lie algebra approach has several merits. First, it takes into account geometrical aspects of the situation. Second, it explains convincingly why it is easy to find exact recursive filters for linear dynamical systems while it is very difficult to filter something like cubic sensor described in the work of Hazewinkel, Marcus and Sussman. The third, and perhaps most important, merit of the Lie algebra approach is the following. As long as the estimation algebra is finite dimensional, not only can the finite dimensional recursive filter be constructed explicitly, but also the filters so constructed is universal in the sense of ChaleyatMaurel and Michel. Moreover, the number of sufficient statistics in the Lie algebra method, which requires computing the conditional probability density, is linear in $n$, where $n$ is the dimension of the state space.

In his talk at the International Congress of Mathematics in 1983, Brockett proposed the problem of classification of all finite-dimensional estimation algebras. Nevertheless, the structure and classification of finite dimensional estimation algebras were studied in detail only since 1990 (cf. [T-W-Y], [Ch-Ya], [Ch-Ya1], [Ch-Ya2], [C-Y-L1], and [C-Y-L2]). Wong introduced the $\Omega$ matrix , which is a matrix whose $(i, j)$ entry is $\omega_{i j}=\frac{\partial f_{j}}{\partial x_{i}}-\frac{\partial f_{i}}{\partial x_{j}}$, where $f$ is the drift term of the state evolution equation.

Recently, Stephen Yau and his co-workers have shown that for any finite dimensional estimation algebra of maximal rank, the $\Omega$-matrix must be constant. After the works of Yau and his co-workers, the main problem of nonlinear filtering becomes: Can one handle nonlinear filtering systems in general beyond the filtering systems constructed by Stephen Yau?

The current paper is to give an affirmative answer to this main problem in nonlinear filtering theory. Our solutions to the nonlinear filtering problems are even better than those classical solutions of Kalman-Bucy for linear filtering with Gaussian initial conditions. First, unlike the classical Kalman-Bucy case which requires real time solution of system of ordinary differential equations of 
dimension $n$, our solution to general nonlinear filtering problem do not need any on-line computation anymore. All computations are reduced to off-line computations. This answers the challenge proposed by Naval Research Office a few years ago: how can one solve the nonlinear filtering problem if adequate amount of computational resources are provided. Second, unlike the classical finite dimensional filters which require the observations for a small time interval (cf. (1.1)), our solution only requires the knowledge of the observation at time $t$.

In section 2, we shall recall some basic concepts and results. In section 3, we shall describe our formula which reduces the nonlinear filtering problem to a off-line computation. In sections 4 and 5 , we give a rigorous proof that our solution converges to the true solution in both pointwise sense and $L^{2}$-sense, respectively.

We gratefully acknowledge the long term support from Army Research Office which plays an important role in our research. We also thank the referee for carefully reading our paper and some useful suggestions to improve the presentation of this paper.

\section{Some basic concepts and results}

In this section, we recall some basic concepts and results from our previous papers. The filtering problem considered here is based on the signal observation model

$$
\left\{\begin{aligned}
d x(t) & =f(x(t)) d t+g(x(t)) d v(t) & & x(0)=x_{0} \\
d y(t) & =h(x(t)) d t+d w(t) & & y(0)=0
\end{aligned}\right.
$$

in which $x, v, y$ and $w$ are respectively $\mathbf{R}^{n}, \mathbf{R}^{p}, \mathbf{R}^{m}$ and $\mathbf{R}^{m}$ valued processes and $v$ and $w$ have components that are independent, standard Brownian processes. We further assume that $n=p ; f$, and $h$ are $C^{\infty}$ smooth vector-valued, and that $g$ is an orthogonal matrix. We shall refer to $x(t)$ as the state of the system at time $t$ and $y(t)$ as the observation at time $t$.

Let $\rho(t, x)$ denote the conditional probability density of the state given the observation $\{y(s): 0 \leq s \leq t\}$. It is well known that $\rho(t, x)$ is given by normalizing a function, $\sigma(t, x)$, which satisfies the following Duncan-Mortensen-Zakai equation:

$$
d \sigma(t, x)=L_{0} \sigma(t, x) d t+\sum_{i=1}^{n} L_{i} \sigma(t, x) d y_{i}(t), \sigma(0, x)=\sigma_{0},
$$

where

$$
L_{0}=\frac{1}{2} \sum_{i=1}^{n} \frac{\partial^{2}}{\partial x_{i}^{2}}-\sum_{i=1}^{n} f_{i} \frac{\partial}{\partial x_{i}}-\sum_{i=1}^{n} \frac{\partial f_{i}}{\partial x_{i}}-\frac{1}{2} \sum_{i=1}^{m} h_{i}^{2},
$$

and for $i=1, \ldots, m, L_{i}$ is the zero degree differential operator of multiplication by $h_{i}$. (Here we have used the notation $p_{i}$ to represent the $i^{\text {th }}$ component of the vector $p) . \sigma_{0}$ is the probability density of the initial point $x_{0}$. 
Equation (2.2) is a stochastic partial differential equation. In real applications, we are interested in constructing robust state estimators from observed sample paths with some property of robustness. Davis studied this problem and proposed some robust algorithms. In our case, his basic idea reduces to defining a new unnormalized density

$$
u(t, x)=\exp \left(-\sum_{i=1}^{m} h_{i}(x) y_{i}(t)\right) \sigma(t, x) .
$$

It is easy to show that $u(t, x)$ satisfies the following time varying partial differential equation

$$
\left\{\begin{aligned}
\frac{\partial u}{\partial t}(t, x) & =L_{0} u(t, x)+\sum_{i=1}^{m} y_{i}(t)\left[L_{0}, L_{i}\right] u(t, x)+ \\
\frac{1}{2} \sum_{i, j=1}^{m} y_{i}(t) y_{j}(t)\left[\left[L_{0}, L_{i}\right], L_{j}\right] u(t, x) &
\end{aligned}\right.
$$

where $[\cdot, \cdot]$ is the Lie bracket as described by the following definition.

It is easy to see (p. 236 of [Ya-Ya1]) that the robust DMZ equation (2.5) is of the form

$$
\begin{aligned}
\frac{\partial u}{\partial t}(t, x)= & \frac{1}{2} \sum_{i=1}^{n} \frac{\partial^{2} u}{\partial x_{i}^{2}}(t, x)-\sum_{i=1}^{n} f_{i}(x) \frac{\partial u}{\partial x_{i}}(t, x)-\sum_{i=1}^{n} \frac{\partial f_{i}}{\partial x_{i}}(x) u(t, x) \\
& -\frac{1}{2} \sum_{i=1}^{m} h_{i}^{2}(x) u(t, x)+\sum_{i=1}^{m} \sum_{j=1}^{n} y_{i}(t) \frac{\partial h_{i}}{\partial x_{j}}(t, x) \frac{\partial u}{\partial x_{j}}(t, x) \\
& +\frac{1}{2} \sum_{i=1}^{m} \sum_{j=1}^{n} y_{i}(t) \frac{\partial^{2} h_{i}}{\partial x_{j}^{2}}(x) u(t, x)-\sum_{i=1}^{m} \sum_{j=1}^{n} y_{i}(t) f_{j}(x) \frac{\partial h_{i}}{\partial x_{j}}(x) u(t, x) \\
& +\frac{1}{2} \sum_{i=1}^{m} \sum_{j=1}^{m} \sum_{k=1}^{n} y_{i}(t) y_{j}(t) \frac{\partial h_{i}}{\partial x_{k}}(x) \frac{\partial h_{j}}{\partial x_{k}}(x) u(t, x) \\
u(0, x)= & \sigma_{0}(x) .
\end{aligned}
$$

\section{Algorithm for real time solution of nonlinear filtering problem without memory}

The fundamental problem of nonlinear filtering theory is how to solve robust D-M-Z equation (2.6) in real time and in memoryless manner. In this section we shall describe our algorithm which achieves this goal for any filtering system with arbitrary initial distribution. Our algorithm is based on the following observation. We should remark that in the following Proposition 3.1 if we replace $\tau_{\ell}$ by $\tau_{\ell-1}$, the same proposition is still true. Therefore we can easily write down left-hand approximation algorithms corresponding to (3.5) if necessary. 
Proposition 3.1. $\widetilde{u}(t, x)$ satisfies the following parabolic equation

$$
\begin{aligned}
& \frac{\partial \widetilde{u}}{\partial t}(t, x)= \\
& \frac{1}{2} \Delta\left(\widetilde{u}(t, x)-\sum_{i=1}^{n} f_{i}(x) \frac{\partial \widetilde{u}}{\partial x_{i}}(t, x)-\left(\sum_{i=1}^{n} \frac{\partial f_{i}}{\partial x_{i}}(x)+\frac{1}{2} \sum_{i=1}^{m} h_{i}^{2}(x)\right) \widetilde{u}(t, x),\right.
\end{aligned}
$$

for $\tau_{\ell-1} \leq t \leq \tau_{\ell}$ if and only if

$$
u(t, x)=e^{-\sum_{i=1}^{m} y_{i}\left(\tau_{\ell}\right) h_{i}(x)} \widetilde{u}(t, x)
$$

satisfies the robust $\mathrm{D}-\mathrm{M}-\mathrm{Z}$ equation with observation being freezed at $y\left(\tau_{\ell}\right)$

$$
\begin{aligned}
& \text { (3.2) } \frac{\partial u}{\partial t}(t, x)=\frac{1}{2} \Delta u(t, x)+\sum_{i=1}^{n}\left(-f_{i}(x)+\sum_{j=1}^{m} y_{j}\left(\tau_{\ell}\right) \frac{\partial h_{j}}{\partial x_{i}}(x)\right) \frac{\partial u}{\partial x_{i}}(t, x) \\
&-\left(\sum_{i=1}^{n} \frac{\partial f_{i}}{\partial x_{i}}(x)+\frac{1}{2} \sum_{i=1}^{m} h_{i}^{2}(x)-\frac{1}{2} \sum_{i=1}^{m} y_{i}\left(\tau_{\ell}\right) \Delta h_{i}(x)+\sum_{i=1}^{m} \sum_{j=1}^{n} y_{i}\left(\tau_{\ell}\right) f_{j}(x) \frac{\partial h_{i}}{\partial x_{j}}(x)\right. \\
&\left.-\frac{1}{2} \sum_{k=1}^{n} \sum_{i=1}^{m} \sum_{j=1}^{m} y_{i}\left(\tau_{\ell}\right) y_{j}\left(\tau_{\ell}\right) \frac{\partial h_{i}}{\partial x_{k}}(x) \frac{\partial h_{j}}{\partial x_{k}}(x)\right) u(t, x) .
\end{aligned}
$$

Proof. This follows by direct computation.

We remark that (3.2) is obtained from D-M-Z equation by freezing the observation term $y(t)$ to $y\left(\tau_{\ell}\right)$. We shall show that the solution of $(3.2)$ approximate the solution of robust D-M-Z equation very well in both pointwise sense and $L^{2}$-sense.

Suppose that $u(t, x)$ is the solution of robust D-M-Z equation and we want to compute $u(\tau, x)$. Let $\mathcal{P}_{k}=\left\{0=\tau_{0}<\tau_{1}<\tau_{2}<\cdots<\tau_{k}=\tau\right\}$ be a partition of $[0, \tau]$. Let $u_{i}(t, x)$ be a solution of the following partial differential equation for $\tau_{i-1} \leq t \leq \tau_{i}$

$$
\left\{\begin{aligned}
\frac{\partial u_{i}}{\partial t}(t, x)= & \frac{1}{2} \Delta u_{i}(t, x) \\
& +\sum_{\ell=1}^{n}\left(-f_{\ell}(x)+\sum_{j=1}^{m} y_{j}\left(\tau_{i}\right) \frac{\partial h_{j}}{\partial x_{\ell}}(x)\right) \frac{\partial u_{i}}{\partial x_{\ell}}(t, x) \\
& -\left(\sum_{\ell=1}^{n} \frac{\partial f_{\ell}}{\partial x_{\ell}}(x)+\frac{1}{2} \sum_{\ell=1}^{m} h_{\ell}^{2}(x)-\frac{1}{2} \sum_{j=1}^{m} y_{j}\left(\tau_{i}\right) \Delta h_{j}(x)\right. \\
& +\sum_{j=1}^{m} \sum_{\ell=1}^{n} y_{j}\left(\tau_{i}\right) f_{\ell}(x) \frac{\partial h_{j}}{\partial x_{\ell}}(x) \\
& \left.-\frac{1}{2} \sum_{p=1}^{n} \sum_{j=1}^{m} \sum_{\ell=1}^{m} y_{j}\left(\tau_{i}\right) y_{\ell}\left(\tau_{i}\right) \frac{\partial h_{j}}{\partial x_{p}}(x) \frac{\partial h_{\ell}}{\partial x_{p}}(x)\right) u_{i}(t, x) \\
u_{i}\left(\tau_{i-1}, x\right)= & u_{i-1}\left(\tau_{i-1}, x\right)
\end{aligned}\right.
$$


Define the norm of the partition $\mathcal{P}_{k}$ by $\left|\mathcal{P}_{k}\right|=\sup _{1 \leq i \leq k}\left\{\left|\tau_{i}-\tau_{i-1}\right|\right\}$.

In section 4 below we shall show that

$$
u(\tau, x)=\lim _{\left|\mathcal{P}_{k}\right| \rightarrow 0} u_{k}\left(\tau_{k}, x\right) .
$$

Therefore it remains to describe an algorithm to compute $u_{k}\left(\tau_{k}, x\right)$. By Proposition $3.1 u_{1}\left(\tau_{1}, x\right)$ can be computed by $\widetilde{u}_{1}\left(\tau_{1}, x\right)$ where $\widetilde{u}_{1}(t, x)$ for $0 \leq t \leq \tau_{1}$ satisfies the following equation

$$
\left\{\begin{aligned}
\frac{\partial \tilde{u}_{1}}{\partial t}(t, x)= & \frac{1}{2} \Delta \widetilde{u}_{1}(t, x)-\sum_{j=1}^{n} f_{j}(x) \frac{\partial \tilde{u}_{1}}{\partial x_{j}}(t, x) \\
& -\left(\sum_{j=1}^{n} \frac{\partial f_{j}}{\partial x_{j}}(x)+\frac{1}{2} \sum_{j=1}^{m} h_{j}^{2}(x)\right) \widetilde{u}_{1}(t, x) \\
\widetilde{u}_{1}(0, x)= & \sigma_{0}(x) \exp \left(\sum_{j=1}^{m} y_{j}\left(\tau_{1}\right) h_{j}(x)\right)
\end{aligned}\right.
$$

In fact,

$$
u_{1}\left(\tau_{1}, x\right)=\exp \left(-\sum_{j=1}^{m} y_{j}\left(\tau_{1}\right) h_{j}(x)\right) \widetilde{u}_{1}\left(\tau_{1}, x\right)
$$

In general Proposition 3.1 tells us that for $i \geq 2, u_{i}\left(\tau_{i}, x\right)$ can be computed by $\widetilde{u}_{i}\left(\tau_{i}, x\right)$ where $\widetilde{u}_{i}(t, x)$ for $\tau_{i-1} \leq t \leq \tau_{i}$ satisfies the following equation

$$
\left\{\begin{aligned}
\frac{\partial \tilde{u}_{i}}{\partial t}(t, x)= & \frac{1}{2} \Delta \widetilde{u}_{i}(t, x)-\sum_{j=1}^{n} f_{j}(x) \frac{\partial \tilde{u}_{i}}{\partial x_{j}}(t, x) \\
& -\left(\sum_{j=1}^{n} \frac{\partial f_{j}}{\partial x_{j}}(x)+\frac{1}{2} \sum_{j=1}^{m} h_{j}^{2}(x)\right) \widetilde{u}_{i}(t, x) \\
\widetilde{u}_{i}\left(\tau_{i-1}, x\right)= & \exp \left(\sum_{j=1}^{m}\left(y_{j}\left(\tau_{i}\right)-y_{j}\left(\tau_{i-1}\right)\right) h_{j}(x)\right) \widetilde{u}_{i-1}\left(\tau_{i-1}, x\right),
\end{aligned}\right.
$$

where the last initial condition comes from

$$
\begin{aligned}
\widetilde{u}_{i}\left(\tau_{i-1}, x\right) & =u_{i}\left(\tau_{i-1}, x\right) \exp \left(\sum_{j=1}^{m} y_{j}\left(\tau_{i}\right) h_{j}(x)\right) \\
& =u_{i-1}\left(\tau_{i-1}, x\right) \exp \left(\sum_{j=1}^{m} y_{j}\left(\tau_{i}\right) h_{j}(x)\right) \\
& =\exp \left(-\sum_{j=1}^{m} y_{j}\left(\tau_{i-1}\right) h_{j}(x)\right) \widetilde{u}_{i-1}\left(\tau_{i-1}, x\right) \exp \left(\sum_{j=1}^{m} y_{j}\left(\tau_{i}\right) h_{j}(x)\right) .
\end{aligned}
$$


In fact,

$$
u_{i}\left(\tau_{i}, x\right)=\exp \left(-\sum_{j=1}^{m} y_{j}\left(\tau_{i}\right) h_{j}(x)\right) \widetilde{u}_{i}\left(\tau_{i}, x\right)
$$

Observe that in our algorithm at step i, we only need the observation at time $\tau_{i-1}$ and $\tau_{i}$. We do not need any other previous observation data. Observe also that the partial differential equation (3.7) is independent of observation $y(t)$. It can be computed off-line. This equation is precisely the equation we dealt with in [Ya-Ya1] of which the fundamental solution is written down explicitly. We shall deal with this problem in a subsequent paper of this one.

\section{Pointwise convergence}

By changing variables from $x_{i}$ to $\sqrt{2} x_{i}$ and by letting

$$
\bar{u}(t, x)=u\left(t, \frac{x}{\sqrt{2}}\right)
$$

we get

$$
\frac{\partial \bar{u}}{\partial t}(t, x)=\frac{\partial u}{\partial t}\left(t, \frac{x}{\sqrt{2}}\right), \quad \begin{aligned}
\frac{\partial \bar{u}}{\partial x_{i}}(t, x) & =\frac{1}{\sqrt{2}} \frac{\partial u}{\partial x_{i}}\left(t, \frac{x}{\sqrt{2}}\right), \\
\frac{\partial^{2} \bar{u}}{\partial x_{i}^{2}}(t, x) & =\frac{1}{2} \frac{\partial^{2} u}{\partial x_{i}^{2}}\left(t, \frac{x}{\sqrt{2}}\right) .
\end{aligned}
$$

Hence the robust D-M-Z equation becomes

$$
\begin{aligned}
\frac{\partial \bar{u}}{\partial t}(t, x)= & \Delta \bar{u}(t, x)+\sum_{i=1}^{n} \sqrt{2}\left[-f_{i}\left(\frac{x}{\sqrt{2}}\right)+\sum_{j=1}^{m} y_{j}(t)\left(\frac{\partial h_{j}}{\partial x_{i}}\right)\left(\frac{x}{\sqrt{2}}\right)\right] \frac{\partial \bar{u}}{\partial x_{i}}(t, x) \\
& -\left[\sum_{i=1}^{n} \frac{\partial f_{i}}{\partial x_{i}}\left(\frac{x}{\sqrt{2}}\right)+\frac{1}{2} \sum_{i=1}^{m} h_{i}^{2}\left(\frac{x}{\sqrt{2}}\right)-\sum_{i=1}^{m} y_{i}(t)\left(\Delta h_{i}\right)\left(\frac{x}{\sqrt{2}}\right)\right. \\
& +\sum_{i=1}^{m} \sum_{j=1}^{n} y_{i}(t) f_{j}\left(\frac{x}{\sqrt{2}}\right) \frac{\partial h_{i}}{\partial x_{j}}\left(\frac{x}{\sqrt{2}}\right) \\
& \left.-\frac{1}{2} \sum_{i, j=1}^{m} \sum_{k=1}^{n} y_{i}(t) y_{j}(t) \frac{\partial h_{i}}{\partial x_{k}}\left(\frac{x}{\sqrt{2}}\right) \frac{\partial h_{j}}{\partial x_{k}}\left(\frac{x}{\sqrt{2}}\right)\right] \bar{u}(t, x) \\
(4.2)= & \Delta \bar{u}(t, x)+\sum_{i=1}^{m} \bar{f}_{i}(t, x) \frac{\partial \bar{u}}{\partial x_{i}}(t, x)-\bar{V}(t, x) \bar{u}(t, x)
\end{aligned}
$$


where

$$
\begin{aligned}
\bar{f}_{i}(t, x)= & \sqrt{2}\left[-f_{i}\left(\frac{x}{\sqrt{2}}\right)+\sum_{j=1}^{m} y_{j}(t)\left(\frac{\partial h_{j}}{\partial x_{i}}\right)\left(\frac{x}{\sqrt{2}}\right)\right] \\
\bar{V}(t, x)= & \sum_{i=1}^{n}\left(\frac{\partial f_{i}}{\partial x_{i}}\right)\left(\frac{x}{\sqrt{2}}\right)+\frac{1}{2} \sum_{i=1}^{m} h_{i}^{2}\left(\frac{x}{\sqrt{2}}\right) \\
& -\sum_{i=1}^{m} y_{i}(t)\left(\Delta h_{i}\right)\left(\frac{x}{\sqrt{2}}\right)+\sum_{i=1}^{m} \sum_{j=1}^{n} y_{i}(t) f_{j}\left(\frac{x}{\sqrt{2}}\right) \frac{\partial h_{i}}{\partial x_{j}}\left(\frac{x}{\sqrt{2}}\right) \\
& -\frac{1}{2} \sum_{i, j=1}^{m} \sum_{k=1}^{n} y_{i}(t) y_{j}(t)\left(\frac{\partial h_{i}}{\partial x_{k}}\right)\left(\frac{x}{\sqrt{2}}\right)\left(\frac{\partial h_{j}}{\partial x_{k}}\right)\left(\frac{x}{\sqrt{2}}\right) .
\end{aligned}
$$

For any $\tau>0$, we shall consider the following parabolic equations on $[0, \tau] \times \mathbf{R}^{n}$

$$
\left\{\begin{aligned}
\frac{\partial \bar{u}}{\partial t}(t, x) & =\Delta \bar{u}(t, x)+\sum_{i=1}^{n} \bar{f}_{i}(t, x) \frac{\partial \bar{u}}{\partial x_{i}}(t, x)-\bar{V}(t, x) \bar{u}(t, x) \\
\bar{u}(0, x) & =\bar{\psi}(x)
\end{aligned}\right.
$$

$$
\left\{\begin{aligned}
\frac{\partial u}{\partial t}(t, x) & =\Delta \widetilde{u}(t, x)+\sum_{i=1}^{n} \widetilde{f}_{i}(\tau, x) \frac{\partial u}{\partial x_{i}}(t, x)-\bar{V}(\tau, x) \widetilde{u}(t, x) \\
\widetilde{u}(0, x) & =\widetilde{\psi}(x)
\end{aligned}\right.
$$

where $\tilde{f}_{i}(\tau, x)$ and $\bar{V}(\tau, x)$ are obtained from $\bar{f}_{i}(t, x)$ and $\bar{V}(t, x)$ by freezing the time variable at $\tau$. We shall assume that $\bar{f}$ and $\widetilde{f}$ grow at most linearly and $\bar{V}$ and $\widetilde{V}$ grow at most quadratically satisfying

$$
\begin{gathered}
|\bar{f}(t, x)| \leq c(1+|x|), \quad|\widetilde{f}(t, x)| \leq c(1+|x|) \\
\left\{\begin{array}{c}
|\nabla \bar{f}(t, x)|=\sqrt{\sum_{i=1}^{n}\left|\nabla \bar{f}_{i}\right|^{2}} \leq c(1+|x|), \\
|\nabla \tilde{f}(\tau, x)|=\sqrt{\sum_{i=1}^{n} \nabla \tilde{f}_{i}} \leq c(1+|x|)
\end{array}\right. \\
|\bar{V}(t, x)| \leq c\left(1+|x|^{2}\right), \quad|\widetilde{V}(\tau, x)| \leq c\left(1+|x|^{2}\right) \\
|\nabla V(t, x)| \leq c(1+|x|), \quad|\nabla \widetilde{V}(\tau, x)| \leq c(1+|x|) .
\end{gathered}
$$

It is clear that if $f(x)$ and its first derivatives, $h(x)$ and its derivatives up to order 3 have linear growth (i.e. $\leq c(1+|x|))$, then $\bar{f}, \widetilde{f}, \bar{V}$ and $\widetilde{V}$ satisfy (4.7)-(4.10) respectively.

In the future paper, we shall treat the case where $h$ has arbitrary polynomial growth. This will include the case where $h$ is a cubic.

The first goal of this section is to prove that if $\widetilde{\psi}(x)$ is close to $\bar{\psi}(x)$ uniformly in $x$, then $\widetilde{u}(\tau, x)$ is close to $\bar{u}(\tau, x)$ uniformly in $x$. From equations (4.5) and 
(4.6), we deduce

$$
\begin{aligned}
\frac{\partial(\bar{u}-\widetilde{u})}{\partial t}= & \Delta(\bar{u}-\widetilde{u})+\sum_{i=1}^{n} \bar{f}_{i} \frac{\partial(\bar{u}-\widetilde{u})}{\partial x_{i}}-\bar{V}(\bar{u}-\widetilde{u}) \\
& +\sum_{i=1}^{n}\left(\bar{f}_{i}-\widetilde{f}_{i}\right) \frac{\partial \widetilde{u}}{\partial x_{i}}-(\bar{V}-\widetilde{V}) \widetilde{u} \\
(4.11)= & (\Delta-\bar{V})(\bar{u}-\widetilde{u})+\sum_{i=1}^{n} \bar{f}_{i} \frac{\partial(\bar{u}-\widetilde{u})}{\partial x_{i}}+\sum_{i=1}^{n}\left(\bar{f}_{i}-\widetilde{f}_{i}\right) \frac{\partial \widetilde{u}}{\partial x_{i}}-(\bar{V}-\widetilde{V}) \widetilde{u} \\
= & (\Delta-\bar{V})(\bar{u}-\widetilde{u})+\sum_{i=1}^{n} \bar{f}_{i} \frac{\partial(\bar{u}-\widetilde{u})}{\partial x_{i}}+G_{\tau}(t, x),
\end{aligned}
$$

where

$$
G_{\tau}(t, x)=\sum_{i=1}^{n}\left(\bar{f}_{i}(t, x)-\widetilde{f}_{i}(\tau, x)\right) \frac{\partial \widetilde{u}}{\partial x_{i}}(t, x)-(\bar{V}(t, x)-\widetilde{V}(\tau, x)) \widetilde{u}(t, x) .
$$

Lemma 4.1: There exists a nonnegative function $\alpha(t, x, y)$ such that

$$
\left\{\begin{aligned}
\frac{\partial \alpha}{\partial t}(t, x, y)= & \Delta_{x} \alpha(t, x, y)-\sum_{i=1}^{n} \bar{f}_{i}(\tau-t, x) \frac{\partial \alpha}{\partial x_{i}}(t, x, y) \\
& -\left[\bar{V}(\tau-t, x)+\sum_{i=1}^{n} \frac{\partial \bar{f}_{i}}{\partial x_{i}}(\tau-t, x)\right] \alpha(t, x, y) \\
\alpha(0, x, y)= & \delta_{y}(x), \quad \int_{x} \alpha(0, x, y) d x=1 .
\end{aligned}\right.
$$

Proof. Let $\beta_{n}(x, y)$ be a sequence of Gaussian with

$$
\int_{x} \beta_{n}(x, y) d x=1 \text { and } \lim _{n \rightarrow \infty} \beta_{n}(x, y)=\delta_{y}(x) .
$$

In view of our previous work [Ya-Ya2], there exists a solution $\alpha_{n}(t, x, y)$ with initial condition $\alpha_{n}(0, x, y)=\beta_{n}(x, y)$. By maximal principle, $\alpha_{n}(t, x, y) \geq 0$ for all $t \geq 0$. We shall take $\alpha(t, x, y)=\lim _{n \rightarrow \infty} \alpha_{n}(t, x, y)$.

Theorem 4.2. Let $w(t, x)=\bar{u}(t, x)-\widetilde{u}(t, x)$ where $\bar{u}$ and $\widetilde{u}$ are the solutions of the parabolic equations (4.5) and (4.6) respectively. Let $\alpha(t, x, y)$ be the nonnegative function in Lemma 4.1. Then

$$
w(\tau, y)=\int_{x} \alpha(\tau, x, y) w(0, x) d x+\int_{0}^{\tau} \int_{x} \alpha(t, x, y) G_{\tau}(t, x) d x
$$

where $G_{\tau}(t, x)$ is given in (4.12). 
Proof.

$$
\begin{aligned}
& \int_{0}^{\tau} \frac{d}{d t} \int_{x} \alpha(\tau-t, x, y) w(t, x) d x= \\
& -\int_{0}^{\tau} \int_{x} \frac{\partial \alpha}{\partial t}(\tau-t, x, y) w(t, x) d x+\int_{0}^{\tau} \int_{x} \alpha(\tau-t, x, y) \frac{\partial w}{\partial t}(t, x) d x
\end{aligned}
$$

L.H.S. of $(4.15)=w(\tau, y)-\int_{x} \alpha(\tau, x, y) w(0, x) d x$

R.H.S. of $(4.15)=-\int_{0}^{\tau} \int_{x} \Delta_{x} \alpha(\tau-t, x, y) w(t, x) d x$

$$
\begin{aligned}
& +\int_{0}^{T} \int_{x} \sum_{i=1}^{n} \bar{f}_{i}(t, x) \frac{\partial \alpha}{\partial x_{i}}(\tau-t, x, y) w(t, x) d x \\
& +\int_{0}^{\tau} \int_{x}\left[\bar{V}(t, x)+\sum_{i=1}^{n} \frac{\partial \bar{f}_{i}}{\partial x_{i}}(t, x)\right] \alpha(\tau-t, x, y) w(t, x) d x \\
& +\int_{0}^{\tau} \int_{x} \alpha(\tau-t, x, y) \frac{\partial w}{\partial t}(t, x) d x \\
= & \int_{0}^{\tau} \int_{x} \alpha(\tau-t, x, y)\left[\frac{\partial w}{\partial t}(t, x)-\Delta w(t, x)\right. \\
& \left.-\sum_{i=1}^{n} \bar{f}_{i}(t, x) \frac{\partial w}{\partial x_{i}}(t, x)+\bar{V}(t, x) w(t, x)\right] d x \\
= & \int_{0}^{\tau} \int_{x} \alpha(\tau-t, x, y) G_{\tau}(t, x) d x, \quad \text { by }(4.13) .
\end{aligned}
$$

In the above computation, we have used the fact proved in [Ya-Ya2] that $\alpha(t, x, y)$ has Gaussian decay in $x$.

Proposition 4.3. Let $\alpha(t, x, y)$ be the nonnegative function in Lemma (4.1). Suppose that $\bar{V}(t, x) \geq-c_{1}$ for some positive constant $c_{1}$. Then

$$
\int_{x} \alpha(\tau, x, y) d x \leq e^{c_{1} \tau}
$$




$$
\begin{aligned}
\text { Proof. } & e^{c_{1} t} \frac{d}{d t}\left(e^{-c_{1} t} \int_{x} \alpha(t, x, y) d x\right)=-c_{1} \int_{x} \alpha(t, x, y) d x+\int_{x} \frac{\partial \alpha}{\partial t}(t, x, y) d x \\
= & -c_{1} \int_{x} \alpha(t, x, y) d x+\int_{x} \Delta_{x} \alpha(t, x, y) d x-\int_{x} \sum_{i=1}^{n} \bar{f}_{i}(\tau-t, x) \frac{\partial \alpha}{\partial x_{i}}(t, x, y) d x \\
& -\int_{x}\left[\bar{V}(\tau-t, x)+\sum_{i=1}^{n} \frac{\partial \bar{f}_{i}}{\partial x_{i}}(\tau-t, x)\right] \alpha(t, x, y) d x \\
= & -c_{1} \int_{x} \alpha(t, x, y) d x-\int_{x} \bar{V}(\tau-t, x) \alpha(t, x, y) d x \\
= & -\int_{x}\left[V(\tau-t, x)+c_{1}\right] \alpha(t, x, y) d x \leq 0
\end{aligned}
$$

It follows that $e^{-c_{1} t} \int_{x} \alpha(t, x, y) d x$ is a decreasing function of $t$ and (4.16) follows.

Theorem 4.4. With the assumption in Proposition 4.3, let $w(t, x)=\bar{u}(t, x)-$ $\widetilde{u}(t, x)$, where $\bar{u}$ and $\widetilde{u}$ are the solutions of the parabolic equations (4.5) and (4.6) respectively. If $\tau$ is small and $w(0, x)$ is small uniformly in $x$, then $w(\tau, x)$ is small uniformly in $x$. More precisely, we have

$$
\sup _{y \in \mathbf{R}^{n}}|w(\tau, y)| \leq e^{c_{1} \tau} \sup _{x \in \mathbf{R}^{n}}|w(0, x)|+\tau e^{c_{1} \tau} \sup _{\substack{x \in \mathbf{R}^{n} \\ 0 \leq t \leq \tau}}\left|G_{\tau}(t, x)\right|
$$

where $G_{\tau}(t, x)$ is given in (4.12).

Proof. In view of (4.5), (4.6) and (4.12), we have

$$
\begin{aligned}
G_{\tau}(t, x)= & \sum_{i=1}^{n}\left(\bar{f}_{i}(t, x)-\tilde{f}_{i}(\tau, x)\right) \frac{\partial \widetilde{u}}{\partial x_{i}}(t, x)-(\bar{V}(t, x)-\widetilde{V}(\tau, x)) \widetilde{u}(t, x) \\
= & \sum_{i=1}^{n} \sqrt{2} \sum_{j=1}^{m}\left(y_{j}(t)-y_{j}(\tau)\right) \frac{\partial h_{j}}{\partial x_{i}}\left(\frac{x}{\sqrt{2}}\right) \frac{\partial \widetilde{u}}{\partial x_{i}}(t, x) \\
& +\left[-\sum_{i=1}^{m}\left(y_{i}(t)-y_{i}(\tau)\right) \Delta h_{i}\left(\frac{x}{\sqrt{2}}\right)\right. \\
& +\sum_{i=1}^{m} \sum_{j=1}^{n}\left(y_{i}(t)-y_{i}(\tau)\right) f_{j}\left(\frac{x}{\sqrt{2}}\right) \frac{\partial h_{i}}{\partial x_{j}}\left(\frac{x}{\sqrt{2}}\right) \\
- & \left.\frac{1}{2} \sum_{i, j=1}^{m} \sum_{k=1}^{n}\left(y_{i}(t) y_{j}(t)-y_{i}(\tau) y_{j}(\tau)\right) \frac{\partial h_{i}}{\partial x_{k}}\left(\frac{x}{\sqrt{2}}\right) \frac{\partial h_{j}}{\partial x_{k}}\left(\frac{x}{\sqrt{2}}\right)\right] \widetilde{u}(t, x) .
\end{aligned}
$$

Therefore if $\tau$ is small, then $G_{\tau}(t, x)$ is uniformly small in $x$ for $0 \leq t \leq \tau$, because both $\widetilde{u}(t, x)$ and $\frac{\partial u}{\partial x_{i}}(t, x)$ have Gaussian decay in $x$ by [Ya-Ya2]. The estimate (4.17) follows readily from Theorem 4.2. 
Now we consider the global situation. For a fixed $T>0$, we want to find the solution $\bar{u}(t, x)$ of the following parabolic equation on $[0, T] \times \mathbf{R}^{n}$

$$
\left\{\begin{aligned}
\frac{\partial \bar{u}}{\partial t}(t, x) & =\Delta \bar{u}(t, x)+\sum_{j=1}^{n} \bar{f}_{j}(t, x) \frac{\partial \bar{u}}{\partial x_{j}}(t, x)-\bar{V}(t, x) \bar{u}(t, x) \\
\bar{u}(0, x) & =\bar{\psi}(x) .
\end{aligned}\right.
$$

Let $\left\{0<\tau_{1}<\tau_{2}<\cdots<\tau_{n}=T\right\}$ be a partition of $[0, T]$. Let $\widetilde{u}_{i}(t, x)$ be the solution of the following parabolic equation on $\left[\tau_{i-1}, \tau_{i}\right] \times \mathbf{R}^{n}$

$$
\left\{\begin{aligned}
\frac{\partial \tilde{u}_{i}}{\partial t}(t, x) & =\Delta \widetilde{u}_{i}(t, x)+\sum_{j=1}^{n} \widetilde{f}_{j}\left(\tau_{i}, x\right) \frac{\partial \tilde{u}_{i}}{\partial x_{j}}(t, x)-\widetilde{V}\left(\tau_{i}, x\right) \widetilde{u}_{i}(t, x) \\
\widetilde{u}_{i}\left(\tau_{i-1}, x\right) & =\widetilde{u}_{i-1}\left(\tau_{i-1}, x\right)
\end{aligned}\right.
$$

where $\widetilde{u}_{1}(0, x)=\bar{\psi}(x), \widetilde{f}_{j}\left(\tau_{i}, x\right)$ and $\widetilde{V}\left(\tau_{i}, x\right)$ are functions independent of $t$ and are equal to $\bar{f}_{j}\left(\tau_{i}, x\right)$ and $\bar{V}\left(\tau_{i}, x\right)$ respectively.

Lemma 4.5. Fix $T$, let $G_{\tau_{i}}(t, x)=\sum_{j=1}^{n}\left(\bar{f}_{j}(t, x)-\tilde{f}_{j}\left(\tau_{i}, x\right)\right) \frac{\partial \tilde{u}_{i}}{\partial x_{j}}(t, x)-(\bar{V}(t, x)-$ $\widetilde{V}(\tau, x)) \widetilde{u}_{i}(t, x)$. For any given $\epsilon>0$, we can choose $n$ sufficiently large so that

$$
\sup _{1 \leq i \leq n} \sup _{\tau_{i-1} \leq t \leq \tau_{i}} \sup _{x \in \mathbf{R}^{n}}\left|G_{\tau_{i}}(t, x)\right| \leq \epsilon .
$$

Proof. This follows from the proof of Theorem 4.4 .

We are now ready to prove the main theorem in this section.

Theorem 4.6. Let $\bar{u}(t, x)$ and $\widetilde{u}_{n}(t, x)$ be the solutions of (4.18) and (4.19) respectively. For any $\epsilon>0$, let $n$ be sufficiently large so that Lemma 4.5 holds. Then

$$
\left|\bar{u}(T, x)-\widetilde{u}_{n}(T, x)\right| \leq \epsilon T e^{c_{1} T},
$$

where $c_{1}$ is the constant in Proposition 4.3.

Proof. In view of $\widetilde{u}_{1}(0, x)=\bar{\psi}(x)=\bar{u}(0, x)$ and Theorem 4.4, we have

$$
\left|\bar{u}\left(\tau_{1}, x\right)-\widetilde{u}_{1}\left(\tau_{1}, x\right)\right| \leq \tau_{1} e^{c_{1} \tau_{1}} \sup _{\substack{x \in \mathbf{R}^{n} \\ 0 \leq t \leq \tau_{1}}}\left|G_{\tau_{1}}(t, x)\right| .
$$

By Theorem 4.4, and induction we have

$$
\begin{aligned}
\left|\bar{u}\left(\tau_{2}, x\right)-\widetilde{u}_{2}\left(\tau_{2}, x\right)\right| \leq & \tau_{1} e^{c_{1} \tau_{1}} e^{c_{1}\left(\tau_{2}-\tau_{1}\right)} \sup _{\substack{x \in \mathbf{R}^{n} \\
0 \leq t \leq \tau_{1}}}\left|G_{\tau_{1}}(t, x)\right| \\
& +\left(\tau_{2}-\tau_{1}\right) e^{c_{1}\left(\tau_{2}-\tau_{1}\right)} \sup _{\substack{x \in \mathbf{R}^{n} \\
\tau_{1} \leq t \leq \tau_{2}}}\left|G_{\tau_{2}}(t, x)\right|
\end{aligned}
$$




$$
\begin{aligned}
&\left|\bar{u}\left(\tau_{n}, x\right)-\widetilde{u}_{n}\left(\tau_{n}, x\right)\right|= \\
& \tau_{1} e^{c_{1} \tau_{n}} \sup _{\substack{x \in \mathbf{R}^{n} \\
0 \leq t \leq \tau_{1}}}\left|G_{\tau_{1}}(t, x)\right|+\left(\tau_{2}-\tau_{1}\right) e^{c_{1}\left(\tau_{n}-\tau_{1}\right)} \sup _{\substack{x \in \mathbf{R}_{n} \\
\tau_{1} \leq t \leq \tau_{2}}}\left|G_{\tau_{2}}(t, x)\right| \\
&+\cdots+\left(\tau_{i}-\tau_{i-1}\right) e^{c_{1}\left(\tau_{n}-\tau_{i-1}\right)} \sup _{\substack{x \in \mathbf{R}^{n} \\
\tau_{i-1} \leq t \leq \tau_{i}}}\left|G_{\tau_{i}}(t, x)\right|+\cdots \\
& \\
&+\left(\tau_{n}-\tau_{n-1}\right) e^{c_{1}\left(\tau_{n}-\tau_{n-1}\right)} \sup _{\substack{x \in \mathbf{R}^{n} \\
\tau_{n-1} \leq t \leq \tau_{n}}}\left|G_{\tau_{n}}(t, x)\right| \\
& \leq \epsilon\left(\tau_{1} e^{c_{1} \tau_{n}}+\left(\tau_{2}-\tau_{1}\right) e^{c_{1}\left(\tau_{n}-\tau_{1}\right)}+\cdots+\left(\tau_{i}-\tau_{i-1}\right) e^{c_{1}\left(\tau_{n}-\tau_{i-1}\right)}+\cdots\right. \\
&\left.+\cdots+\left(\tau_{n}-\tau_{n-1}\right) e^{c_{1}\left(\tau_{n}-\tau_{n-1}\right)}\right) \\
& \leq \epsilon\left[\tau_{1}+\left(\tau_{2}-\tau_{1}\right)+\cdots+\left(\tau_{i}-\tau_{i-1}\right)+\cdots+\left(\tau_{n}-\tau_{n-1}\right)\right] e^{c_{1} T} \\
&= \epsilon T e^{c_{1} T} .
\end{aligned}
$$

Theorem 4.7. Fix $T>0$, let $\mathcal{P}_{n}=\left\{0<\tau_{1}<\tau_{2}<\cdots<\tau_{n}=T\right\}$ be a partition of $[0, T]$. Let $\bar{u}(t, x)$ be the solution of the following parabolic equation on $[0, T] \times \mathbf{R}^{n}$

$$
\left\{\begin{aligned}
\frac{\partial \bar{u}}{\partial t}(t, x) & =\Delta \bar{u}(t, x)+\sum_{j=1}^{n} \bar{f}_{j}(t, x) \frac{\partial \bar{u}}{\partial x_{j}}(t, x)-\bar{V}(t, x) \bar{u}(t, x) \\
\bar{u}(0, x) & =\psi(x) .
\end{aligned}\right.
$$

Let $\widetilde{u}_{i}(t, x)$ be the solution of the following parabolic equation on $\left[\tau_{i-1}, \tau_{i}\right] \times \mathbf{R}^{n}$

$$
\left\{\begin{aligned}
\frac{\partial \tilde{u}_{i}}{\partial t}(t, x) & =\Delta \widetilde{u}_{i}(t, x)+\sum_{j=1}^{n} \tilde{f}_{j}\left(\tau_{i}, x\right) \frac{\partial \tilde{u}_{i}}{\partial x_{j}}(t, x)-\widetilde{V}\left(\tau_{i}, x\right) \widetilde{u}_{i}(t, x) \\
\widetilde{u}_{i}\left(\tau_{i-1}, x\right) & =\widetilde{u}_{i-1}\left(\tau_{i-1}, x\right) .
\end{aligned}\right.
$$

where $\widetilde{u}_{1}(0, x)=\psi(x)$ and $\widetilde{f}_{j}\left(\tau_{i}, x\right)=\bar{f}_{j}\left(\tau_{i}, x\right), \widetilde{V}\left(\tau_{i}, x\right)=\bar{V}\left(\tau_{i}, x\right)$ are obtained from $\bar{f}_{j}(t, x)$ and $\bar{V}(t, x)$ by freezing time variable at $\tau_{i}$. Then

$$
\bar{u}(\tau, x)=\lim _{\left|\mathcal{P}_{n}\right| \rightarrow 0} \widetilde{u}_{n}\left(\tau_{n}, x\right) \text { uniformly in } x .
$$




\section{5. $L^{2}$-convergence}

In section 4 we have shown that the solution $\widetilde{u}(t, x)$ of $(4.6)$ is uniformly close to the solution $\bar{u}(t, x)$ of (4.5) for $0 \leq t \leq \tau$ if $\tau$ is sufficiently small and $\widetilde{\psi}(x)=\widetilde{u}(0, x)$ is uniformly close to $\bar{\psi}(x)=\bar{u}(0, x)$. In this section, we shall show that $\widetilde{u}(t, x)$ is also close to $\bar{u}(t, x)$ in $L^{2}$ sense. We first recall the following lemma.

Lemma 5.1. If $\frac{d \alpha}{d t}(t) \leq c \alpha(t)+\beta(t)$, where $c$ is constant, then $e^{-c t} \alpha(t)-\alpha(0) \leq$ $\int_{0}^{t} e^{-c s} \beta(s) d s$.

Let $\bar{f}_{2 R}, \widetilde{f}_{2 R}, \bar{V}_{2 R}$ and $\widetilde{V}_{2 R}$ be the functions obtained by multiplying $\bar{f}, \widetilde{f}, \bar{V}$ and $\widetilde{V}$ respectively by a cut off function $\sigma$ which is equal to one in the ball of radius $R \geq 1$ and equal to zero outside a ball of radius $2 R$. We can choose $\sigma$ such that

$$
|\nabla \sigma(x)| \leq \frac{4}{1+|x|} \text { and }|\Delta \sigma(x)| \leq \frac{4}{1+|x|^{2}}
$$

Consider the following equations

$$
\begin{aligned}
\frac{\partial \bar{u}_{2 R}}{\partial t} & =\Delta \bar{u}_{2 R}+\sum_{i=1}^{n}\left(\bar{f}_{2 R}\right)_{i} \frac{\partial \bar{u}_{2 R}}{\partial x_{i}}-\bar{V}_{2 R} \bar{u}_{2 R} \\
\frac{\partial \widetilde{u}_{2 R}}{\partial t} & =\Delta \widetilde{u}_{2 R}+\sum_{i=1}^{n}\left(\widetilde{f}_{2 R}\right)_{i} \frac{\partial \widetilde{u}_{2 R}}{\partial x_{i}}-\widetilde{V}_{2 R} \widetilde{u}_{2 R}
\end{aligned}
$$

in the ball $B_{2 R}$ of radius $2 R$ with the Neumann condition, where $\left(f_{2 R}\right)_{i}$ and $\left(\widetilde{f}_{2 R}\right)_{i}$ denote the $i^{\text {th }}$ components of $f_{2 R}$ and $\widetilde{f}_{2 R}$ respectively. Let $\bar{\psi}_{2 R}(x)=$ $\bar{\psi}(x) \sigma(x)$ and $\widetilde{\psi}_{2 R}(x)=\widetilde{\psi}(x) \sigma(x)$ to be the initial conditions of (5.2) and (5.3) respectively. Then (5.2) and (5.3) have unique solutions respectively for $t \in$ $[0, \infty)$ with Neuman condition on $\partial B_{2 R} \times(0, T]$.

Lemma 5.2. Assume that (4.7)-(4.10) hold and $|\Delta \bar{f}(t, x)| \leq c(1+|x|)$, $|\Delta \widetilde{f}(t, x)| \leq c(1+|x|)$ where $c \geq 4$ is a constant. Let $\widetilde{c}$ and $\delta$ be positive constants such that $\widetilde{c}:=\widetilde{c}+\delta<\frac{5}{254}$. Choose $\tau$ and $\epsilon$ suitably small with $\tau+\epsilon<\delta$. Then the following conclusions (i), (ii) and (iii) hold for any $0 \leq t \leq \tau$ for both $\rho \in\{\bar{\rho}, \widetilde{\rho}\}, u \in\{\bar{u}, \widetilde{u}\}$, and where $\bar{\rho}(t, x)=\frac{\widetilde{c}\left(1+|x|^{2}\right)}{t+\epsilon}, \widetilde{\rho}(t, x)=\frac{\widetilde{\widetilde{c}}\left(1+|x|^{2}\right)}{t+\epsilon}$, 
(i) $\int_{\{t\} \times B_{2 R}} e^{\bar{\rho}} \bar{u}_{2 R}^{2} \leq \int_{\{0\} \times B_{2 R}} e^{\bar{\rho}} \bar{u}_{2 R}^{2}$

(ii) $\int_{\{t\} \times B_{2 R}} e^{\bar{\rho}}\left|\nabla \bar{u}_{2 R}\right|^{2} \leq \int_{\{0\} \times B_{2 R}} e^{\bar{\rho}}\left|\nabla \bar{u}_{2 R}\right|^{2}+\int_{0}^{t} \int_{B_{2 R}} e^{\bar{\rho}(x, s)}\left|\bar{u}_{2 R}(s, x)\right|^{2}$

(iii) $\int_{\{t\} \times B_{2 R}} e^{\bar{\rho}}\left|\Delta \bar{u}_{2 R}\right|^{2} \leq \int_{\{0\} \times B_{2 R}} e^{\bar{\rho}}\left|\Delta \bar{u}_{2 R}\right|^{2}$

$$
\begin{aligned}
& +O\left(\int_{[0, t] \times B_{2 R}} e^{\bar{\rho}}|\nabla \bar{\rho}|^{2}\left|\bar{f}_{2 R}\right|^{2}\left|\nabla \bar{u}_{2 R}\right|^{2}+\int_{[0, t] \times B_{2 R}} e^{\bar{\rho}}\left|\nabla \bar{f}_{2 R}\right|^{2}\left|\nabla \bar{u}_{2 R}\right|^{2}\right. \\
& +\int_{[0, t] \times B_{2 R}} e^{\bar{\rho}}\left|\bar{f}_{2 R}\right|\left|\nabla \bar{u}_{2 R}\right|^{2}\left|\Delta \bar{f}_{2 R}\right|+\int_{[0, t] \times B_{2 R}} e^{\bar{\rho}}\left|\bar{f}_{2 R}\right|^{4}\left|\nabla \bar{u}_{2 R}\right|^{2} \\
& \left.+\int_{[0, t] \times B_{2 R}} e^{\bar{\rho}}\left|\nabla\left(\bar{V}_{2 R} \bar{u}_{2 R}\right)\right|^{2}+\int_{[0, t] \times B_{2 R}} e^{\bar{\rho}}\left|\nabla \bar{u}_{2 R}\right|^{2}\left(\sum_{i=1}^{n} \frac{\partial\left(\bar{f}_{2 R}\right)_{i}}{\partial x_{i}}\right)^{2}\right) .
\end{aligned}
$$

Moreover the following inequalities hold for both $\{\bar{\rho}, \bar{f}, \bar{V}\}$, or $\{\bar{\rho}, \widetilde{f}, \widetilde{V}\}$, or $\{\widetilde{\rho}, \bar{f}, \bar{V}\}$ or $\{\widetilde{\rho}, \widetilde{f}, \widetilde{V}\}$ if $\delta$ is small enough.

(iv) $\frac{\partial \bar{\rho}}{\partial t}+2|\nabla \bar{\rho}|^{2}-\sum_{i=1}^{n} \bar{f}_{i} \frac{\partial \bar{\rho}}{\partial x_{i}}-\sum_{i=1}^{n} \frac{\partial \bar{f}_{i}}{\partial x_{i}}-2 \bar{V} \leq 0$.

Proof. (i), (ii) and (iii) follow from Lemma 1.3 of [Ya-Ya2] by setting $\epsilon_{1}=\frac{1}{5}$ in that lemma. Inequality (iv) follows from

$$
\begin{aligned}
\frac{\partial \widetilde{\rho}}{\partial t}+2|\nabla \widetilde{\rho}|^{2}-\sum_{i=1}^{n} \bar{f}_{i} \frac{\partial \widetilde{\rho}}{\partial x_{i}} & -\sum_{i=1}^{n} \frac{\partial \bar{f}_{i}}{\partial x_{i}}-2 \bar{V} \\
& \leq\left[\frac{-\widetilde{\widetilde{c}}(1-8 \tilde{\widetilde{c}})}{(t+\epsilon)^{2}}+\frac{2 c \tilde{\widetilde{c}}}{t+\epsilon}+(n+2) c\right](1+|x|)^{2} .
\end{aligned}
$$

as $1-8 \approx \widetilde{c} \geq 0$ 
Proposition 5.3. Consider the parabolic differential equations (4.5) and (4.6). Let $\phi$ be any smooth function defined on $\mathbf{R}^{n}$ with compact support contained in a domain $\Omega$. Let $\bar{\rho}$ be any smooth function on $\mathbf{R}_{+} \times \mathbf{R}^{n}$ satisfying

$$
\frac{\partial \bar{\rho}}{\partial t}+2|\nabla \bar{\rho}|^{2}-\sum_{i=1}^{n} \bar{f}_{i} \frac{\partial \bar{\rho}}{\partial x_{i}}-\sum_{i=1}^{n} \frac{\partial \bar{f}_{i}}{\partial x_{i}}-2 \bar{V} \leq 0
$$

Then

$$
\begin{aligned}
& \quad \frac{d}{d t} \int_{\{t\} \times \Omega} \phi^{2} e^{\bar{\rho}}(\bar{u}-\widetilde{u})^{2} \\
& \leq \quad \int_{\{t\} \times \Omega} \phi^{2} e^{\bar{\rho}}(\bar{u}-\widetilde{u})^{2}+10 \int_{\{t\} \times \Omega} e^{\bar{\rho}(\bar{u}-\widetilde{u})^{2}|\nabla \phi|^{2}} \\
& \quad+4 \int_{\{t\} \times \Omega} e^{\bar{\rho} \mid}\left|\sum_{i=1}^{n} \bar{f}_{i} \frac{\partial \phi}{\partial x_{i}}\right|^{2}(\bar{u}-\widetilde{u})^{2} \\
& \quad+4 \int_{\{t\} \times \Omega} e^{\bar{\rho}} \phi^{2}\left|\sum_{i=1}^{n}\left(\bar{f}_{i}-\widetilde{f}_{i}\right) \frac{\partial \rho}{\partial x_{i}}\right|^{2} \widetilde{u}^{2} \\
& \quad+2 \int_{\{t\} \times \Omega} e^{\bar{\rho}} \phi^{2} \widetilde{u}|\bar{f}-\widetilde{f}|^{2}+4 \int_{\{t\} \times \Omega} e^{\bar{\rho}} \phi^{2} \widetilde{u}^{2}|\bar{V}-\widetilde{V}|^{2} \\
& \quad+2 \int_{\{t\} \times \Omega} e^{\bar{\rho}} \phi^{2}|\bar{f}-\widetilde{f}|^{2} \widetilde{u}^{2}+4 \int_{\{t\} \times \Omega} e^{\bar{\rho}} \phi^{2} \widetilde{u}^{2}\left|\sum_{i=1}^{n}\left(\frac{\partial \bar{f}_{i}}{\partial x_{i}}-\frac{\partial \widetilde{f}_{i}}{\partial x_{i}}\right)\right|^{2}
\end{aligned}
$$

Proof. From equations (4.5) and (4.6) we deduce

$$
\begin{aligned}
& \frac{\partial(\bar{u}-\widetilde{u})}{\partial t}= \\
& \Delta(\bar{u}-\widetilde{u})+\sum_{i=1}^{n} f_{i} \frac{\partial(\bar{u}-\widetilde{u})}{\partial x_{i}}-V(u-\widetilde{u})+\sum_{i=1}^{n}\left(\bar{f}_{i}-\widetilde{f}_{i}\right) \frac{\partial \widetilde{u}}{\partial x_{i}}-(\bar{V}-\widetilde{V}) \widetilde{u}
\end{aligned}
$$


Then using (5.6) and integrating by part, we obtain

$$
\begin{aligned}
& \frac{d}{d t} \int_{\{t\} \times \Omega} \phi^{2}(\bar{u}-\widetilde{u})^{2} e^{\bar{\rho}} \\
& \leq \int_{\{t\} \times \Omega} e^{\bar{\rho}} \phi^{2}(\bar{u}-\widetilde{u})^{2}\left(\frac{\partial \bar{\rho}}{\partial t}+2|\nabla \bar{\rho}|^{2}-\sum_{i=1}^{n} \bar{f}_{i} \bar{\rho}_{i}-\sum_{i=1}^{n} \frac{\partial \bar{f}_{i}}{\partial x_{i}}-2 \bar{V}\right) \\
& -\frac{1}{2} \int_{\{t\} \times \Omega} e^{\bar{\rho}} \phi^{2}|\nabla(\bar{u}-\widetilde{u})|^{2}+8 \int_{\{t\} \times \Omega} e^{\bar{\rho}}(\bar{u}-\widetilde{u})^{2}|\nabla \phi|^{2} \\
& -2 \int_{\{t\} \times \Omega} \phi e^{\bar{\rho}}\left(\sum_{i=1}^{n} \bar{f}_{i} \phi_{i}\right)(\bar{u}-\widetilde{u})^{2} \\
& -4 \int_{\{t\} \times \Omega} e^{\bar{\rho}} \phi\left[\sum_{i=1}^{n}\left(\bar{f}_{i}-\widetilde{f}_{i}\right) \phi_{i}\right] \widetilde{u}(\bar{u}-\widetilde{u}) \\
& -2 \int_{\{t\} \times \Omega} e^{\bar{\rho}} \phi^{2} \sum_{i=1}^{n}\left(\bar{f}_{i}-\widetilde{f}_{i}\right) \frac{\partial \bar{\rho}}{\partial x_{i}} \widetilde{u}(\bar{u}-\widetilde{u})+2 \int_{\{t\} \times \Omega} e^{\bar{\rho}} \phi^{2} \widetilde{u}^{2}|f-\widetilde{f}|^{2} \\
& -2 \int_{\{t\} \times \Omega} e^{\bar{\rho}} \phi^{2}(\bar{u}-\widetilde{u}) \widetilde{u} \sum_{i=1}^{n}\left(\frac{\partial \bar{f}_{i}}{\partial x_{i}}-\frac{\partial \widetilde{f}_{i}}{\partial x_{i}}\right) \\
& -2 \int_{\{t\} \times \Omega} e^{\bar{\rho}} \phi^{2}(\bar{u}-\widetilde{u}) \widetilde{u}(\bar{V}-\widetilde{V}) .
\end{aligned}
$$

In view of (5.4), (5.7) implies

$$
\begin{aligned}
& \frac{d}{d t} \int_{\{t\} \times \Omega} \phi^{2} e^{\bar{\rho}}(\bar{u}-\widetilde{u})^{2} \\
& \leq 8 \int_{\{t\} \times \Omega} e^{\bar{\rho}}(\bar{u}-\widetilde{u})^{2}|\nabla \phi|^{2}+4 \int_{\{t\} \times \Omega} e^{\bar{\rho}}(\bar{u}-\widetilde{u})^{2}\left|\sum_{i=1}^{n} \bar{f}_{i} \frac{\partial \phi}{\partial x_{i}}\right|^{2} \\
& +\frac{1}{4} \int_{\{t\} \times \Omega} e^{\bar{\rho}} \phi^{2}(\bar{u}-\widetilde{u})^{2}+\frac{1}{4} \int_{\{t\} \times \Omega} e^{\bar{\rho}} \phi^{2}(\bar{u}-\widetilde{u})^{2} \\
& +4 \int_{\{t\} \times \Omega} e^{\bar{\rho}} \phi^{2}\left|\sum_{i=1}^{n}\left(\bar{f}_{i}-\widetilde{f}_{i}\right) \frac{\partial \bar{\rho}}{\partial x_{i}}\right|^{2} \widetilde{u}^{2}+2 \int_{\{t\} \times \Omega} e^{\bar{\rho}} \phi^{2} \widetilde{u}^{2}|\bar{f}-\widetilde{f}|^{2} \\
& +\frac{1}{4} \int_{\{t\} \times \Omega} e^{\bar{\rho}} \phi^{2}(\bar{u}-\widetilde{u})^{2}+4 \int_{\{t\} \times \Omega} e^{\bar{\rho}} \phi^{2} \widetilde{u}^{2}|\bar{V}-\widetilde{V}|^{2} \\
& +4\left[\frac{1}{2} \int_{\{t\} \times \Omega} e^{\bar{\rho}}(\bar{u}-\widetilde{u})^{2}|\nabla \phi|^{2}+\frac{1}{2} \int_{\{t\} \times \Omega} e^{\bar{\rho}} \phi^{2}|\bar{f}-\widetilde{f}|^{2} \widetilde{u}^{2}\right] \\
& +2\left[\frac{1}{8} \int_{\{t\} \times \Omega} e^{\bar{\rho}} \phi^{2}(\bar{u}-\widetilde{u})^{2}+2 \int_{\{t\} \times \Omega} e^{\bar{\rho}} \phi^{2} \widetilde{u}^{2}\left|\sum_{i=1}^{n}\left(\frac{\partial \bar{f}_{i}}{\partial x_{i}}-\frac{\partial \widetilde{f}_{i}}{\partial x_{i}}\right)\right|^{2}\right] .
\end{aligned}
$$

Inequality (5.5) follows immediately. 
The following theorem states when $\tau$ is sufficiently small and $\bar{\psi}(x)$ is close to $\widetilde{\psi}(x)$ in $L^{2}$-sense, then the solution $\widetilde{u}(t, x)$ of (4.6) approximates the solution $\bar{u}(t, x)$ of $(4.5)$ well in $L^{2}$-sense.

Theorem 5.4. Consider the parabolic differential equations (4.5) and (4.6). Assume that

$$
\begin{aligned}
&|\bar{f}(t, x)| \leq c(1+|x|),|\nabla \bar{f}(t, x)| \leq c,|\Delta \bar{f}(t, x)| \leq c \\
&|\widetilde{f}(t, x)| \leq c(1+|x|),|\nabla \tilde{f}(t, x)| \leq c,|\Delta \widetilde{f}(t, x)| \leq c \\
&|\bar{V}(t, x)| \leq c\left(1+|x|^{2}\right),|\nabla \bar{V}(t, x)| \leq c(1+|x|) \\
&|\widetilde{V}(t, x)| \leq c\left(1+|x|^{2}\right),|\nabla \widetilde{V}(t, x)| \leq c(1+|x|),
\end{aligned}
$$

where $c \geq 4$ is a constant. Let $\widetilde{c}$ and $\delta$ be positive constants such that $\widetilde{c}:=\widetilde{c}+\delta<$ $\frac{5}{254}$. Let

Suppose that

$$
\bar{\rho}(t, x)=\frac{\widetilde{c}\left(1+|x|^{2}\right)}{t+\epsilon}, \quad \widetilde{\rho}(t, x)=\frac{\widetilde{\widetilde{c}}\left(1+|x|^{2}\right)}{t+\epsilon} .
$$

$$
\begin{aligned}
& \int_{\mathbf{R}^{n}} e^{\bar{\rho}(0, x)}\left(|\bar{\psi}(x)|^{2}+|\nabla \bar{\psi}(x)|^{2}+|\Delta \bar{\psi}(x)|^{2}\right)<\infty, \\
& \int_{\mathbf{R}^{n}} e^{\tilde{\rho}(0, x)}\left(|\widetilde{\psi}(x)|^{2}+|\nabla \widetilde{\psi}(x)|^{2}+|\Delta \widetilde{\psi}(x)|^{2}\right)<\infty .
\end{aligned}
$$

Choose $\tau$ and $\epsilon$ suitably small so that $\tau+\epsilon<\delta$ and the conclusions of Lemma 5.2 hold. Suppose that for $0 \leq t \leq \tau$

$$
\begin{aligned}
|\bar{f}(t, x)-\tilde{f}(t, x)| & \leq \widetilde{\epsilon}_{1} c(1+|x|) \\
\left|\sum_{i=1}^{n}\left(\frac{\partial \bar{f}_{i}}{\partial x_{i}}(t, x)-\frac{\partial \tilde{f}_{i}}{\partial x_{i}}(t, x)\right)\right| & \leq \widetilde{\epsilon}_{1} c \\
|\bar{V}(t, x)-\widetilde{V}(t, x)| & \leq \widetilde{\epsilon}_{1} c\left(1+|x|^{2}\right) \\
\int_{\mathbf{R}^{n}} e^{\bar{\rho}(0, x)}|\bar{\psi}(x)-\widetilde{\psi}(x)|^{2} & \leq \widetilde{\epsilon}_{2} .
\end{aligned}
$$

Then

$$
\begin{aligned}
\int_{\{t\} \times \mathbf{R}^{n}} e^{\bar{\rho}}(\bar{u}-\widetilde{u})^{2} & \leq \tilde{\epsilon}_{2} e^{t}+16 \widetilde{\epsilon}_{1}^{2} c^{2} \widetilde{c}^{2} \frac{t}{\epsilon(t+\epsilon)} e^{t} d_{1}+24 t \widetilde{\epsilon}_{1}^{2} c^{2} e^{t} d_{1} \\
& \leq \widetilde{\epsilon}_{2} e^{\tau}+\widetilde{\epsilon}_{1}^{2} \tau e^{\tau} c_{1}
\end{aligned}
$$

where $d_{1}=\int_{\mathbf{R}^{n}} e^{\tilde{\rho}(0, x)}(\widetilde{\psi}(x))^{2}$ and $c_{1}=\frac{16 c^{2} \widetilde{c}^{2} d_{1}}{\epsilon^{2}}+24 c^{2} d_{1}$.

Proof. Let $R_{0} \geq 1$ and $B_{R_{0}}^{c}=\left\{x \in \mathbf{R}^{n}:|x|>R_{0}\right\}$ and

$$
\phi(x)=\left\{\begin{array}{cl}
1 & \text { for }|x| \leq R_{0} \\
\frac{\log R-\log |x|}{\log R-\log R_{0}} & \text { for } R_{0} \leq|x| \leq R \\
0 & \text { for }|x| \geq R
\end{array}\right.
$$


Let $\Omega$ be defined as $B_{R}$ in Proposition 5.3. In view of Lemma 5.1 and (5.5), we have

$$
\begin{aligned}
& e^{-t} \int_{\{t\} \times \Omega} \phi^{2} e^{\bar{\rho}}(\bar{u}-\widetilde{u})^{2}-\int_{\{0\} \times \Omega} \phi^{2} e^{\bar{\rho}}(\bar{u}-\widetilde{u})^{2} \\
& \leq 10 \int_{0}^{t} e^{-s} \int_{\{s\} \times \Omega} e^{\bar{\rho}}(\bar{u}-\widetilde{u})^{2}|\nabla \phi|^{2} \\
& +4 \int_{0}^{t} e^{-s} \int_{\{s\} \times \Omega} e^{\rho}\left|\sum_{i=1}^{n} \bar{f}_{i} \frac{\partial \phi}{\partial x_{i}}\right|^{2}(\bar{u}-\widetilde{u})^{2} \\
& +4 \int_{0}^{t} e^{-s} \int_{\{s\} \times \Omega} e^{\bar{\rho}} \phi^{2}\left|\sum_{i=1}^{n}\left(\bar{f}_{i}-\widetilde{f}_{i}\right) \frac{\partial \rho}{\partial x_{i}}\right|^{2} \widetilde{u}^{2} \\
& +2 \int_{0}^{t} e^{-s} \int_{\{s\} \times \Omega} e^{\bar{\rho}} \phi^{2} \widetilde{u}^{2}|\bar{f}-\widetilde{f}|^{2} \\
& -4 \int_{0}^{t} e^{-s} \int_{\{s\} \times \Omega} e^{\bar{\rho}} \phi^{2} \widetilde{u}^{2}|\bar{V}-\widetilde{V}|^{2}+2 \int_{0}^{t} e^{-s} \int_{\{s\} \times \Omega} e^{\bar{\rho}} \phi^{2}|\bar{f}-\widetilde{f}|^{2} \widetilde{u}^{2} \\
& +4 \int_{0}^{t} e^{-s} \int_{\{s\} \times \Omega} e^{\bar{\rho}} \phi^{2} \widetilde{u}^{2}\left|\sum_{i=1}^{n}\left(\frac{\partial \bar{f}_{i}}{\partial x_{i}}-\frac{\partial \tilde{f}_{i}}{\partial x_{i}}\right)\right|^{2} . \\
& \leq \frac{10}{\left(\log R-\log R_{0}\right)^{2}} \int_{0}^{t} e^{-s} \int_{\{s\} \times B_{R_{0}}^{c}} e^{\bar{\rho}}(\bar{u}-\widetilde{u})^{2} \\
& +\frac{16 c^{2}}{\left(\log R-\log R_{0}\right)^{2}} \int_{0}^{t} e^{-s} \int_{\{s\} \times B_{R_{0}}^{c}} e^{\bar{\rho}}(\bar{u}-\widetilde{u})^{2} \\
& +4 \int_{0}^{t} e^{-s} \int_{\{s\} \times \Omega} e^{\bar{\rho}} \phi^{2}\left|\sum_{i=1}^{n}\left(\bar{f}_{i}-\widetilde{f}_{i}\right) \frac{\partial \bar{\rho}}{\partial x_{i}}\right|^{2} \widetilde{u}^{2} \\
& +4 \int_{0}^{t} e^{-s} \int_{\{s\} \times \Omega} e^{\bar{\rho}} \phi^{2} \widetilde{u}^{2}|\bar{f}-\widetilde{f}|^{2} \\
& +4 \int_{0}^{t} e^{-s} \int_{\{s\} \times \Omega} e^{\bar{\rho}} \phi^{2} \widetilde{u}^{2}|\bar{V}-\widetilde{V}|^{2} \\
& +4 \int_{0}^{t} e^{-s} \int_{\{s\} \times \Omega} e^{\bar{\rho}} \phi^{2} \widetilde{u}^{2}\left|\sum_{i=1}^{n}\left(\frac{\partial \bar{f}_{i}}{\partial x_{i}}-\frac{\partial \tilde{f}_{i}}{\partial x_{i}}\right)\right|^{2}
\end{aligned}
$$

Observe that (5.11) implies

$$
e^{t} \int_{\{0\} \times B_{R}} e^{\bar{\rho}}(\bar{u}-\widetilde{u})^{2} \leq \widetilde{\epsilon}_{2} e^{t} .
$$


By Corollary 4.1 of [Ya-Ya2], $u$ and $\widetilde{u}$ decay like Gaussian in $x$ variables. So we shall assume

$$
\max _{x \in \mathbf{R}^{n}}(|u|,|\widetilde{u}|) \leq D_{1} e^{-D_{2}|x|^{2}} \quad \text { for } t \text { small }
$$

for some $D_{1}, D_{2}>0$. In view of the proof of Corollary 4.1 of [Ya-Ya2], we can take $2 D_{2} \geq \frac{\widetilde{c}}{\epsilon}+1$ for sufficiently small $t$.

$$
\begin{aligned}
& \frac{e^{t}\left(10+16 c^{2}\right)}{\left(\log R-\log R_{0}\right)^{2}} \int_{0}^{t} \int_{\{s\} \times B_{R_{0}}^{c}} e^{\bar{\rho}}(\bar{u}-\widetilde{u})^{2} \\
\leq & \frac{4 t e^{t} D_{1}^{2}\left(10+16 c^{2}\right)}{\left(\log R-\log R_{0}\right)^{2}} c \exp \left(-R_{0}^{2}+\frac{\widetilde{c}}{\epsilon}\right) .
\end{aligned}
$$

Recall that $|\nabla \bar{\rho}|^{2}=\frac{4 \tilde{c}^{2}|x|^{2}}{(t+\epsilon)^{2}}$. Hence (5.8) implies

$$
\begin{aligned}
& 4 e^{t} \int_{0}^{t} \int_{\{0\} \times B_{R}} e^{\bar{\rho}}|\bar{f}-\widetilde{f}|^{2}|\nabla \bar{\rho}|^{2} \widetilde{u}^{2} \\
\leq \quad & 16 \widetilde{\epsilon}_{1}^{2} c^{2} \widetilde{c}^{2} e^{t} \int_{0}^{t} \frac{1}{(s+\epsilon)^{2}} \int_{\{s\} \times B_{R}} e^{\tilde{\rho}} \widetilde{u}^{2} .
\end{aligned}
$$

Similarly we can prove that

$$
e^{t} \int_{0}^{t} \int_{\{s\} \times B_{R}} e^{\bar{\rho}} \widetilde{u}^{2}|\bar{f}-\widetilde{f}|^{2} \leq 4 e^{t} \widetilde{\epsilon}_{1}^{2} c^{2} \int_{0}^{t} \int_{\{s\} \times B_{R}} e^{\tilde{\rho}} \widetilde{u}^{2}
$$

$$
4 e^{t} \int_{0}^{t} \int_{\{s\} \times B_{R}} e^{\bar{\rho}} \widetilde{u}^{2}|\bar{V}-\widetilde{V}|^{2} \leq 4 e^{t} \widetilde{\epsilon}_{1}^{2} c^{2} \int_{0}^{t} \int_{\{s\} \times B_{R}} e^{\tilde{\rho}} \widetilde{u}^{2}
$$

and

$$
\begin{aligned}
& 4 e^{t} \int_{0}^{t} \int_{\{s\} \times B_{R}} e^{\bar{\rho}} \widetilde{u}^{2}\left|\sum_{i=1}^{n}\left(\frac{\partial \bar{f}_{i}}{\partial x_{i}}-\frac{\partial \widetilde{f}_{i}}{\partial x_{i}}\right)\right|^{2} \\
\leq & 4 e^{t} \widetilde{\epsilon}_{1}^{2} c^{2} \int_{0}^{t} \int_{\{s\} \times B_{R}} e^{\bar{\rho}} \widetilde{u}^{2} .
\end{aligned}
$$


Hence

$$
\begin{aligned}
& \int_{\{t\} \times B_{R_{0}}} e^{\bar{\rho}}(\bar{u}-\widetilde{u})^{2} \leq \widetilde{\epsilon}_{2} e^{t}+\frac{4 t e^{t} D_{1}^{2}\left(10+16 c^{2}\right)}{\left(\log R-\log R_{0}\right)^{2}} c e^{\left(-R_{0}^{2}+\frac{\tilde{c}}{\epsilon}\right)} \\
& +16 \widetilde{\epsilon}_{1}^{2} c^{2} \widetilde{c}^{2} e^{t} \int_{0}^{t} \frac{1}{(s+\epsilon)^{2}} \int_{\{s\} \times B_{R}} e^{\tilde{\rho}} \widetilde{u}^{2}+16 e^{t} \widetilde{\epsilon}_{1}^{2} c^{2} \int_{0}^{t} \int_{\{s\} \times B_{R}} e^{\tilde{\rho}} \widetilde{u}^{2} \\
& +4 e^{t} \widetilde{\epsilon}_{1}^{2} c^{2} \int_{0}^{t} \int_{\{s\} \times B_{R}} e^{\tilde{\rho}} \widetilde{u}^{2}+4 e^{t} \widetilde{\epsilon}_{1}^{2} c^{2} \int_{0}^{t} \int_{\{s\} \times B_{R}} e^{\tilde{\rho} \widetilde{u}^{2}} \\
& \leq \widetilde{\epsilon}_{2} e^{t}+16 \widetilde{\epsilon}_{1}^{2} c^{2} \widetilde{c}^{2} e^{t} \int_{0}^{t} \frac{1}{(s+\epsilon)^{2}} \int_{\{0\} \times \mathbf{R}^{n}} e^{\tilde{\rho}} \widetilde{u}^{2} \\
& +\frac{4 t e^{t} D_{1}^{2}\left(10+16 c^{2}\right)}{\left(\log R-\log R_{0}\right)^{2}} c e^{\left(-R_{0}^{2}+\frac{\tilde{\tau}}{\epsilon}\right)}+24 e^{t} \widetilde{\epsilon}_{1}^{2} c^{2} \int_{0}^{t} \int_{\{0\} \times \mathbf{R}^{n}} e^{\tilde{\rho}} \widetilde{u}^{2} \\
& \leq \widetilde{\epsilon}_{2} e^{t}+\frac{16 \widetilde{\epsilon}_{1}^{2} c^{2} \widetilde{c}^{2} e^{t} d_{1} t}{\epsilon(t+\epsilon)}+24 t e^{t} \widetilde{\epsilon}_{1}^{2} c^{2} d_{1}+\frac{4 t e^{t} D_{1}^{2}\left(10+16 c^{2}\right)}{\left(\log R-\log R_{0}\right)^{2}} c e^{\left(-R_{0}^{2}+\frac{\tilde{c}}{\epsilon}\right)} .
\end{aligned}
$$

Let $R_{0}$ go to infinity in (5.20), we obtain the estimate in the statement of Theorem 5.4.

Now we are ready to consider the global situation. For a fixed $T>0$, we want to find the solution $\bar{u}(t, x)$ of $(4.5)$.

Theorem 5.5. Let $\bar{u}(t, x)$ and $\widetilde{u}_{k}(t, x)$ be the solutions of (4.18) and (4.19) respectively. For $\widetilde{\epsilon}_{1}>0$, let $\left|\mathcal{P}_{k}\right|=\sup _{i}\left\{\left|t_{i}-t_{i-1}\right|\right\}$ be sufficiently small so that the following estimates hold.

$$
\begin{aligned}
\left|\bar{f}(t, x)-\tilde{f}\left(\tau_{i}, x\right)\right| & \leq \widetilde{\epsilon}_{1} c(1+|x|), \text { for } \tau_{i-1} \leq t \leq \tau_{i} \\
\left|\sum_{j=1}^{n}\left(\frac{\partial \bar{f}_{j}}{\partial x_{j}}(t, x)-\frac{\partial \widetilde{f}_{j}}{\partial x_{j}}\left(\tau_{i}, x\right)\right)\right| & \leq \widetilde{\epsilon}_{1} c \\
\left|\bar{V}(t, x)-\widetilde{V}\left(\tau_{i}, x\right)\right| & \leq \widetilde{\epsilon}_{1} c\left(1+|x|^{2}\right) .
\end{aligned}
$$

Then

$$
\int_{\mathbf{R}^{n}} e^{\bar{\rho}(T, x)}\left(\bar{u}(T, x)-\widetilde{u}_{k}(T, x)\right)^{2} \leq \widetilde{\epsilon}_{1}^{2} c_{1} k\left|\mathcal{P}_{k}\right| e^{T} \leq \widetilde{\epsilon}_{1}^{2} c_{1} c_{2}(T)
$$

where $\bar{\rho}(t, x)=\frac{\widetilde{c}\left(1+|x|^{2}\right)}{t+\epsilon}$ so that the conclusion of Theorem 5.4 holds, $c_{1}$ is the constant in Theorem 5.4 and $c_{2}(T)$ is a constant depends only on $T$.

Proof. In view of $\widetilde{u}_{1}(0, x)=\psi(x)=\bar{u}(0, x)$ and Theorem 5.4, we have

$$
\begin{aligned}
& \int_{\left\{\tau_{1}\right\} \times \mathbf{R}^{n}} e^{\bar{\rho}}(\bar{u}-\widetilde{u})^{2} \leq \widetilde{\epsilon}_{1}^{2} \tau_{1} e^{\tau_{1}} c_{1} \\
& \int_{\left\{\tau_{2}\right\} \times \mathbf{R}^{n}} e^{\bar{\rho}}(\bar{u}-\widetilde{u})^{2} \leq \widetilde{\epsilon}_{1}^{2} c_{1}\left[\tau_{1} e^{\tau_{2}}+\left(\tau_{2}-\tau_{1}\right) e^{\tau_{2}-\tau_{1}}\right] .
\end{aligned}
$$


By Theorem 5.4 and induction, we have

$$
\begin{aligned}
\int_{\left\{\tau_{k}\right\} \times \mathbf{R}^{n}} e^{\bar{\rho}}(\bar{u}-\widetilde{u})^{2}= & \widetilde{\epsilon}_{1}^{2} c_{1}\left[\tau_{1} e^{\tau_{k}}+\left(\tau_{2}-\tau_{1}\right) e^{\tau_{k}-\tau_{1}}+\left(\tau_{3}-\tau_{2}\right) e^{\tau_{k}-\tau_{2}}\right. \\
& \left.+\cdots+\left(\tau_{k}-\tau_{k-1}\right) e^{\tau_{k}-\tau_{k-1}}\right] \\
\leq & \widetilde{\epsilon}_{1}^{2} c_{1} k\left|\mathcal{P}_{k}\right| e^{T} \\
\leq & \widetilde{\epsilon}_{1}^{2} c_{1} c_{2}(T) .
\end{aligned}
$$

As a consequence of Theorem 5.6, we have the following $L^{2}$-convergent theorem.

Theorem 5.6. Fix $T>0$, let $\mathcal{P}_{k}=\left\{0<\tau_{1}<\tau_{2}<\cdots<\tau_{k}=T\right\}$ be a partition of $[0, T]$. Let $\bar{u}(t, x)$ be the solution of $(4.5)$ on $[0, T] \times \mathbf{R}^{n}$. Let $\widetilde{u}_{i}(t, x)$ be the solution of (4.19) on $\left[\tau_{i-1}, \tau_{i}\right] \times \mathbf{R}^{n}$. Let $\bar{\rho}(t, x)=\frac{\widetilde{c}\left(1+|x|^{2}\right)}{t+\epsilon}$ so that the conclusion of Theorem 5.5 holds. Then

$$
\lim _{\left|\mathcal{P}_{k}\right| \rightarrow 0} \int_{\{T\} \times \mathbf{R}^{n}} \bar{\rho}\left(u-\widetilde{u}_{k}\right)^{2}=0 .
$$

By the method of [Ya-Ya2], it is not hard to give a pointwise estimate of $e^{\bar{\rho}}(\bar{u}-\widetilde{u})^{2}$ which means that there is a pointwise estimate of $(\bar{u}-\widetilde{u})^{2}$ with decay in space.

\section{References}

[Ch] J. Chen, On uniquity of Yau filters, Proceedings of the American Control Conference (Baltimore, Maryland), June 1994, 252-254.

[Ch-Ya1] J. Chen and S. S.-T. Yau, Finite-dimensional filters with nonlinear drift VI: Linear structure of $\Omega$, Math. Control Signals Systems 9 (1996), 370-385.

[Ch-Ya2] _ Finite-dimensional filters with nonlinear drift VII: Mitter conjecture and structure of $\eta$, SIAM J. Control Optim. 35 (1997), 1116-1131.

[C-Y-L1] J. Chen, Stephen S.-T. Yau, and C. W. Leung, Finite-dimensional filters with nonlinear drift IV: Classification of finite-dimensional estimation algebras of maximal rank with state space dimension 3, SIAM J. Control Optim. 34 (1996), 179-198.

[C-Y-L2] _ Finite-dimensional filters with nonlinear drift VIII: Classification of finitedimensional estimation algebras of maximal rank with state-space dimension 4, SIAM J. Control Optim. 35 (1997), 1132-1141.

[Ch-Ya] W. L. Chiou and S. S.-T. Yau, Finite-dimensional filters with nonlinear drift II: Brockett's problem on classification of finite-dimensional estimation algebras, SIAM J. Control Optim. 32 (1994), 297-310.

[Fl-Mi] W. H. Fleming and S. K. Mitter, Optimal control and nonlinear filtering for non degenerate diffusion processes, Stochastics 8 (1982), 63-77.

[Hu-Ya] G. W. Hu and S. S.-T. Yau, Finite dimensional filters with nonlinear drift X: Explicit solution of DMZ equation, IEEE Trans. Automat. Control, to appear.

[Ya-Hu] G.-Q. Hu and S. S.-T. Yau, Finite dimensional filters with nonlinear drift XIV: Classification of finite dimensional estimation algebras of maximal rank with arbitrary state space dimension and Mitter conjecture, Preprint.

[Ma] S. Marcus, Algebraic and geometric methods in nonlinear filtering, SIAM J. Control Optim. 22 (1984), 817-844. 
[T-W-Y] L. F. Tam, W.S. Wong, and S. S.-T. Yau, On a necessary and sufficient condition for finite dimensionality of estimation algebras, SIAM J. Control Optim. 28 (1990), 173-185.

[Ya-Ya1] S. T. Yau and S. S.-T. Yau, Finite dimensional filters with nonlinear drift III: Duncan-Mortensen-Zakai equation with arbitrary initial condition for Kalman-Bucy filtering system and Benes filtering system, IEEE Transactions on Aerospace and Electronic Systems 33 (1997), 1277-1294.

[Ya-Ya2] _ Existence and uniqueness and decay estimates for the time dependent parabolic equation with application to Duncan-Mortensen-Zakai equation, Asian J. Math. 2 (1998), 1079-1149.

Department of Mathematics, Harvard University, Cambridge, MA 02138.

E-mail address: yau@math.harvard.edu

Department of Mathematics, Statistics, and Computer Science (M/C 249), University of Illinois at Chicago, 851 South Morgan Street, Chicago, IL 60607-7045, U.S.A.

E-mail address: yau@uic.edu 